\title{
Resistance to thyroid hormone-beta co-existing with partially empty sella in a Jordanian male
}

\author{
Ohoud Al Mohareb', Mussa H Al Malki', O Thomas Mueller² and Imad Brema1 \\ 1Obesity, Endocrine and Metabolism Centre, King Fahad Medical City, Riyadh, Kingdom of Saudi Arabia and \\ 2Department of Pathology and Laboratory Medicine, Molecular and Biochemical Section, All Children Hospital, St \\ Petersburg, Florida, USA
}

Correspondence should be addressed

to I Brema

Email

ibrema@kfmc.med.sa

\section{Summary}

Resistance to thyroid hormone-beta (RTHbeta) is a rare inherited syndrome characterized by variable reduced tissue responsiveness to the intracellular action of triiodothyronine (T3), the active form of the thyroid hormone. The presentation of RTHbeta is quite variable and mutations in the thyroid hormone receptor beta $(T H R-B)$ gene have been detected in up to $90 \%$ of patients. The proband was a 34-year-old Jordanian male who presented with intermittent palpitations. His thyroid function tests (TFTs) showed a discordant profile with high free T4 (FT4) at 45.7 pmol/L (normal: 12-22), high free T3 (FT3) at $11.8 \mathrm{pmol} / \mathrm{L}$ (normal: 3.1-6.8) and inappropriately normal TSH at $3.19 \mathrm{mIU} / \mathrm{L}$ (normal: $0.27-4.2$ ). Work up has confirmed normal alpha subunit of TSH of $0.1 \mathrm{ng} / \mathrm{mL}$ (normal $<0.5$ ) and pituitary MRI showed no evidence of a pituitary adenoma; however, there was an interesting coincidental finding of partially empty sella. RTHbeta was suspected and genetic testing confirmed a known mutation in the THR-B gene, where a heterozygous A to $G$ base change substitutes valine for methionine at codon 310. Screening the immediate family revealed that the eldest son (5 years old) also has discordant thyroid function profile consistent with RTHbeta and genetic testing confirmed the same M310V mutation that his father harbored. Moreover, the 5-year-old son had hyperactivity, impulsivity and aggressive behavior consistent with attention deficit hyperactivity disorder (ADHD). This case demonstrates an unusual co-existence of RTHbeta and partially empty sella in the same patient which, to our knowledge, has not been reported before.

\section{Learning points:}

- We report the coincidental occurrence of RTHbeta and a partially empty sella in the same patient that has not been previously reported.

- TFTs should be done in all children who present with symptoms suggestive of ADHD as RTHbeta is a common finding in these children.

- The management of children with ADHD and RTHbeta could be challenging for both pediatricians and parents and the administration of T3 with close monitoring may be helpful in some cases.

- Incidental pituitary abnormalities do exist in patients with RTHbeta, although extremely rare, and should be evaluated thoroughly and separately. 


\section{Background}

This is the first report of an individual (the proband) with RTHbeta associated with an incidentally diagnosed partially empty sella on pituitary MRI. We acknowledge that there is no direct etiological or pathological link between the two conditions. The association between RTHbeta and ADHD constitutes a diagnostic and management dilemma. It would be interesting to assess the energy expenditure level in cases of RTHbeta and ADHD (such as in our second case) and compare that to cases of RTHbeta without ADHD, to verify the findings of Weiss et al. (1) who described a large case series of RTHbeta in children who were shown to have increased energy expenditure levels.

\section{Case presentation}

The proband was a 34-year-old Jordanian male who complained of intermittent palpitations for 5 months prior to his presentation to the cardiology clinic. He was prescribed bisoprolol $2.5 \mathrm{mg}$ once daily and was then referred to our endocrine clinic for further assessment. Apart from his presenting complaint, he had no other symptoms of hyperthyroidism, such as excessive sweating, tremors, weight loss or disturbed sleep. He was unaware of any family history of thyroid dysfunction. He had good energy levels, normal libido and physical examination revealed a slightly overweight male with weight $82 \mathrm{~kg}$, height $172 \mathrm{~cm}$ and BMI $27.4 \mathrm{~kg} /$ $\mathrm{m}^{2}$, with a regular pulse ( 72 beats per minute) and blood pressure of $120 / 82 \mathrm{mmHg}$; he had no palpable goiter and no evidence of thyroid eye disease. He had normal body physique, muscle mass and body hair. Physical examination of the other systems was unremarkable. The proband has two sons who were 5 and 3 years old. Further history from the parents revealed that the 5-year-old son had symptoms of hyperactivity, impulsivity, restlessness and aggressive behavior for the past 2 years. His growth rate has been satisfactory with a body weight of $22 \mathrm{~kg}$ (between 75th and 90th centiles) and height of $110 \mathrm{~cm}$ (between 50th and 75th centiles), as shown in the growth chart in Fig. 1. This child was a product of full term pregnancy and a normal vaginal delivery. He had no dysmorphic features; however, initial psychiatric evaluation suggested the diagnosis of ADHD. The parents reported no complaints or concerns about the 3-year-old son.

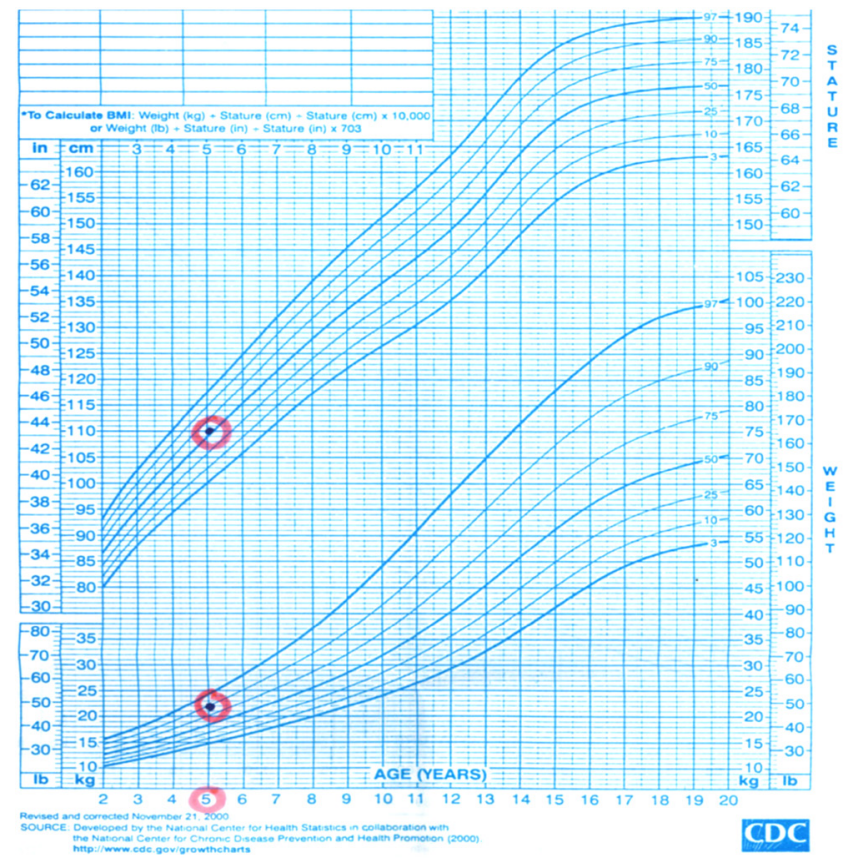

Figure 1

Growth chart of the 5-year-old son with RTH due to M310V mutation.

\section{Investigation}

The proband underwent assessment at the cardiology clinic with electrocardiogram or ECG which was normal and TFTs, which showed a discordant profile with high free T4 (FT4) at $45.7 \mathrm{pmol} / \mathrm{L}$ (normal range: 12-22), high free T3 (FT3) at $11.8 \mathrm{pmol} / \mathrm{L}$ (normal range: 3.1-6.8) and a TSH $3.19 \mathrm{mIU} / \mathrm{L}$ (normal range: 0.27-4.2). Further work up of the proband confirmed a normal alpha subunit level of $0.1 \mathrm{ng} / \mathrm{mL}$ (normal <0.5) and normal SHBG of $20.4 \mathrm{nmol} / \mathrm{L}$ (normal reference range: 10-60), thereby excluding hepatic sensitivity to high thyroid hormone levels. Thyroid auto-antibody screen was negative. MRI of the pituitary did not show a pituitary adenoma; however, there was an incidental finding of a partially empty sella (Fig. 2). Further biochemical evaluation of the proband in a mid-morning serum sample revealed normal levels of other anterior pituitary hormones as follows: cortisol $406 \mathrm{nmol} / \mathrm{L}(08: 00 \mathrm{~h}$ reference $>500)$, prolactin: $123 \mathrm{mIU} / \mathrm{L}$ (normal range: $86-324$ ), IGF-1: $26 \mathrm{nmol} / \mathrm{L}$ (normal range: 14.2-36.9), total testosterone: $9.3 \mathrm{nmol} / \mathrm{L}$ (normal range: 8.6-29), LH: 2.4 IU/L, FSH 1.6 IU/L. DNA sequence analysis of the proband's thyroid hormone receptor beta gene (THR-B) confirmed a known missense mutation in Exon 7, where a heterozygous A to $G$ base change substitutes valine for methionine at codon 310 (Fig. 3). 

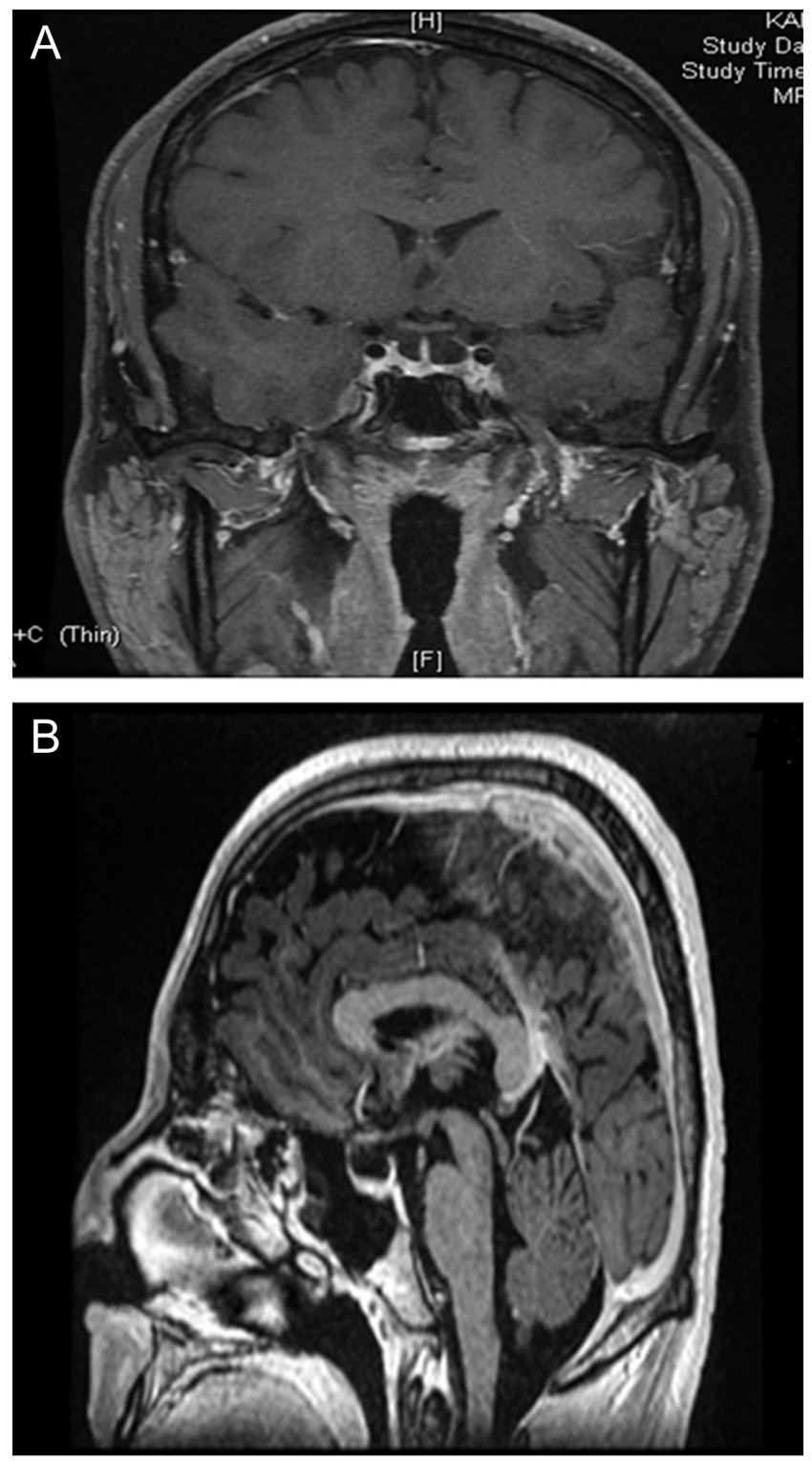

Figure 2

Coronal (A) and sagittal (B) sections of the MRI pituitary showing incidental partially empty sella in the proband.

The proband's eldest son (5 years old) also had discordant TFTs with a very high FT4 at $46.4 \mathrm{pmol} / \mathrm{L}$ (normal range: 12-22), high FT3 at $16.5 \mathrm{pmol} / \mathrm{L}$ (normal range: 2.8-7.1) and inappropriately normal TSH of $4.3 \mathrm{mIU} / \mathrm{L}$ (normal range: 0.85-6.5), consistent with RTHbeta. DNA sequencing of the THR-B gene, confirmed the presence of the same mutation as his father.

The proband's 3-year-old son had no clinical or biochemical phenotype to suggest RTHbeta and has tested negative for this mutation.

\section{Treatment}

The proband was prescribed bisoprolol $2.5 \mathrm{mg}$, which he initially took on daily basis for several months but then was less compliant with this treatment and took it on intermittent basis.

The 5-year child with RTHbeta and ADHD was referred to the pediatric endocrinology and childhood psychiatry departments as he is out of our scope of service. There is some evidence presented by Weiss et al. in a small study that supraphysiological doses of liothyronine (L-T3) in patients with ADHD and RTHbeta may improve hyperactivity and impulsivity in some of the patients compared to those with ADHD alone; however, the number of patients in that study was very small and further larger studies in larger number of patients are needed.

\section{Outcome and follow-up}

The proband took his treatment on an intermittent basis although he still complains of palpitations. The 5-year-old child was referred to pediatric endocrinology; however, his father opted for observation without pharmacological treatment as he was concerned about side effects of medications.

\section{Discussion}

We present a Jordanian family with RTHbeta due to a missense mutation (M310V) in the THR-B gene, which has been previously reported in very few cases, by Mitchell et al. and Amor et al. Our two cases represent a significant addition to the literature on this mutation $(2,3)$.

Sakurai et al. described the first mutation causing RTHbeta in 1989 (4). According to recent reports (5), there are approximately 170 mutations that are listed in the Human Gene Mutation Database, to date. For logistic reasons, we could not carry out functional studies, however, this mutation is a well-recognized pathological mutation as previously reported $(2,3)$. Furthermore, missense variant functionality programs (such as Sorting Intolerant Form Tolerant and Polyphon) indicate that this is a highly conserved amino acid suggesting a change would be deleterious. This is further supported by the clinical phenotype of RTH in the proband and the affected son. Interestingly, the four cases of M310V previously described by Mitchell and colleagues were part of a large group of RTHbeta cases that included 54 adults and 13 

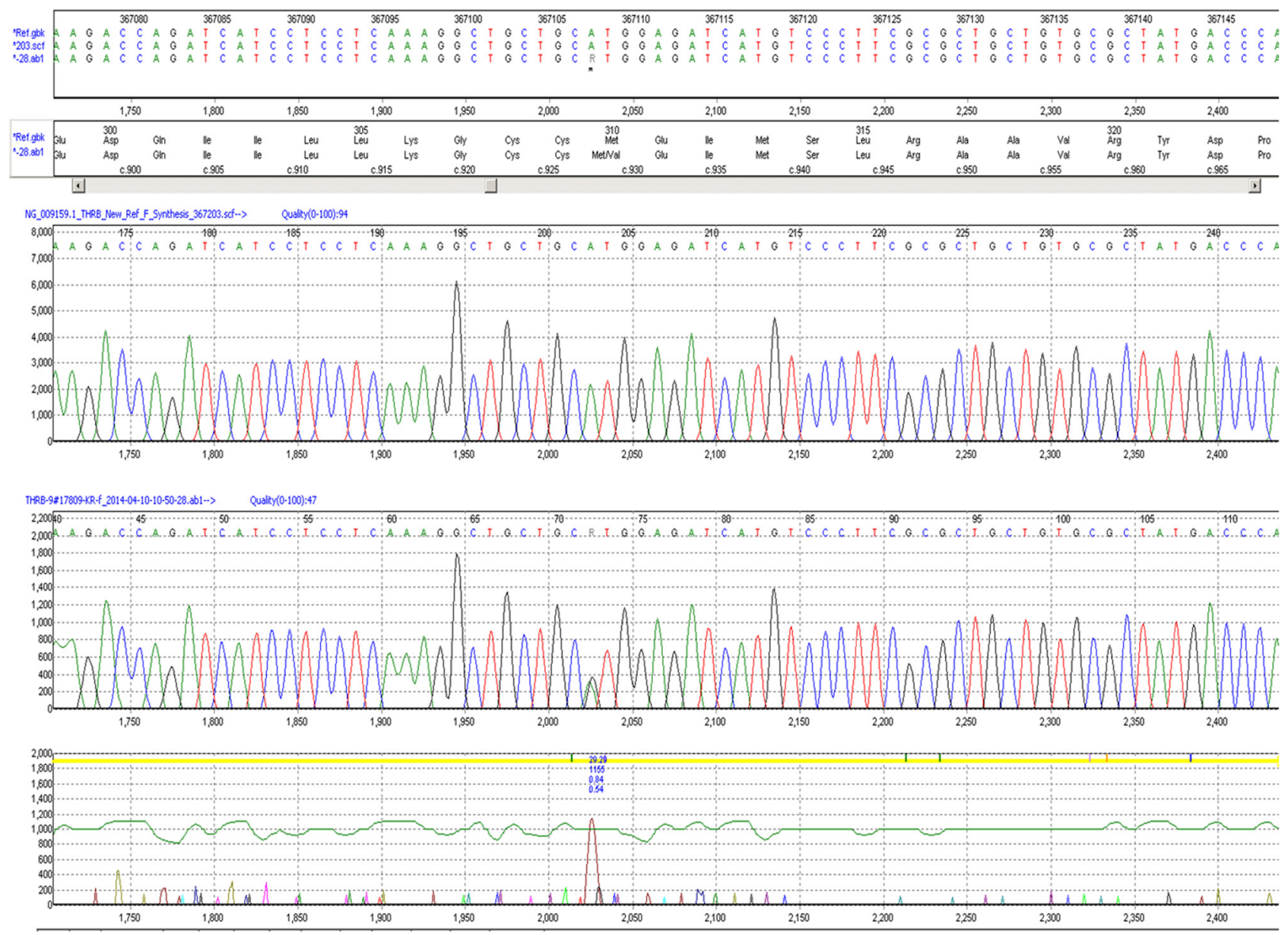

Figure 3

Sequence analysis of the thyroid hormone receptor B gene in the proband (forward and reverse).

children from 13 unrelated families with 25 different mutations in THR-B and the authors clearly showed markedly increased energy expenditure and food intake in both adults and children (2). However, the number of RTHbeta cases due to M310V mutation was too small in that cohort.

The proband presented at the age of 34 years with intermittent palpitations, which responded well to Bisoprolol for a few months; however, the patient became less compliant with his treatment despite the occurrence of occasional symptoms. The proband had no palpable goiter (which occurs in 55-65\% of patients of RTHbeta) and no other physical abnormalities on examination.

The presence of a partially empty sella together with RTH is probably an incidental finding; however, to our knowledge, this finding has not been described previously in cases of RTHbeta. Furthermore, the patient did not complain of headaches or visual symptoms. He had no history of a head injury and there was no clinical suspicion of raised intracranial hypertension. Reassuringly, levels of all anterior pituitary hormones including prolactin, IGF-1 and cortisol were normal, especially as it is recognized that up to $10 \%$ of patients with primary empty sella may have endocrinopathies, the commonest abnormalities being hyperprolactinemia and hypopituitarism (6). Although serum testosterone level was on the lower end of the normal range; nonetheless, the patient denied any symptoms of hypogonadism and was clinically eugonadal. Radiological and biochemical follow-up would be a reasonable approach in this patient as progression from partial to complete empty sella has been documented with hormonal abnormalities such as hypopituitarism in some cases. The association between RTHbeta and pituitary abnormalities is extremely rare with only one reported case of RTHbeta and coincidental pituitary incidentaloma (7).

The 5-year-old affected son presented from early childhood with an extreme phenotype of hyperactivity, 
restlessness, aggressive behavior, interrupted sleep, hyperimpulsivity and difficulty at school. These features are quite suggestive of $\mathrm{ADHD}$ and this patient was referred for evaluation by a child psychiatrist to confirm this disorder, which is a well-recognized presentation of RTH in childhood $(1,8,9,10)$. The feedback from initial childhood psychiatric evaluation was supportive of the diagnosis of ADHD. Weiss and colleagues reported in a small study that some children with both ADHD and RTHbeta may respond to supraphysiological doses of liothyronine (L-T3), which has been shown to reduce both the hyperactivity and impulsivity in some but not all of these children, compared to those without RTHbeta. However, the number of children who were studied was extremely small making it difficult to draw any clear cut conclusions and larger studies are needed to confirm these findings (1). The growth of this child, as shown in Fig. 3, looks normal; however, it may be affected in future. Therefore, this patient was referred to the pediatric endocrine department for further evaluation and possible treatment as deemed necessary and his response to treatment may be presented at a later stage as it may take some time. Moran et al. recently reported a case of a 9-year-old Saudi child with RTHbeta due to a homozygous THR-B mutation resulting in cardiac dysfunction that was successfully treated with the combination of triiodothyroacetic acid (TRIAC) and carbimazole (11). The authors suggested that if the beneficial effects of the combination of TRIAC and carbimazole persisted long term, this combination can be used in heterozygous cases of RTHbeta as well.

The extreme variability in clinical phenotype despite having the same mutation is well known in cases of RTHbeta and has been well documented in the literature across families and within the same family; however, the etiology of this variation remains unknown. The proband has five brothers and two sisters, some of whom had children, however, we were unable to screen the full family due to logistic and geographical reasons as they reside outside Saudi Arabia; however, we strongly advised family screening, especially for children whose growth and development may be negatively affected by their functional thyroid status.

In conclusion, we describe an interesting family with RTHbeta due to a known M310V mutation in which the proband presented with palpitations yet coincidentally had a partially empty sella. To our knowledge, this has not been previously reported. The proband's 5-year-old son had RTHbeta due to the same mutation that his father harbored and had a phenotype of ADHD, which is a challenging association with RTHbeta in children.

\section{Declaration of interest}

The authors declare that there is no conflict of interest that could be perceived as prejudicing the impartiality of the research reported.

\section{Funding}

King Fahad Medical City Research Fund.

\section{Patient consent}

Written informed consent was obtained from the proband for publication. The proband also signed guardian consent for his two sons' genetic tests.

\section{Author contribution statement}

$\mathrm{O}$ Al Mohareb is the named physician of the proband and has significantly contributed to the manuscript writing. I Brema is the senior and corresponding author and has contributed significantly to the manuscript writing. $\mathrm{M} \mathrm{H} \mathrm{Al} \mathrm{Malki} \mathrm{is} \mathrm{a} \mathrm{co-author} \mathrm{and} \mathrm{contributed} \mathrm{to} \mathrm{manuscript} \mathrm{writing.}$ O T Mueller is a co-author and did the genetic sequencing and contributed to the manuscript writing. We are very grateful to Dr Sara G I Suliman, consultant endocrinologist at Imperial College London Diabetes Centre, Abu Dhabi, for her valuable contribution on proofreading and editing.

\section{References}

1 Weiss RE, Stein MA \& Refetoff S. Behavioral effects of liothyronine (L-T3) in children with attention deficit hyperactivity disorder in the presence and absence of resistance to thyroid hormone. Thyroid 1997 7 389-393. (https://doi.org/10.1089/thy.1997.7.389)

2 Mitchell CS, Savage DB, Dufour S, Schoenmakers N, Murgatroyd P, Befroy D, Halsall D, Northcott S, Raymond-Barker P, Curran S, et al. Resistance to thyroid hormone is associated with raised energy expenditure, muscle mitochondrial uncoupling, and hyperphagia. Journal of Clinical Investigation 2010120 1345-1354. (https://doi. org/10.1172/JCI38793)

3 Amor AJ, Halperin I, Alfayate R, Borras VM, Escribano A, Gonzalez C, Gutirrez A, Mauri M, Perez P, Picomicron A, et al. Identification of four novel mutations in the thyroid hormone receptor-beta gene in 164 Spanish and 2 Greek patients with resistance to thyroid hormone. Hormones 201413 74-78. (https://doi.org/10.1007/ BF03401322)

4 Sakurai A, Takeda K, Ain K, Ceccarelli P, Nakai A, Seino S, Bell GI, Refetoff S \& DeGroot LJ. Generalized resistance to thyroid hormone associated with a mutation in the ligand-binding domain of the human thyroid hormone receptor beta. PNAS 198986 8977-8981. (https://doi.org/10.1073/pnas.86.22.8977)

5 Dumitrescu AM \& Refetoff S. The syndromes of reduced sensitivity to thyroid hormone. Biochimica et Biophysica Acta 20131830 3987-4003. (https://doi.org/10.1016/j.bbagen.2012.08.005)

6 De Marinis L, Bonadonna S, Bianchi A, Maira G \& Giustina A. Primary empty sella. Journal of Clinical Endocrinology and Metabolism 200590 5471-5477. (https://doi.org/10.1210/jc.2005-0288)

7 Sriphrapradang C, Srichomkwun P, Refetoff S and Mamanasiri S. A novel thyroid hormone receptor beta gene mutation (G251V) in a Thai patient with resistance to thyroid hormone coexisting with 
pituitary incidentaloma. Thyroid 201626 1804-1806. (https://doi. org/10.1089/thy.2016.0450)

8 Rohrer T, Gassmann K, Pohlenz J \& Dorr HG. Resistance to thyroid hormone - goiter and attention deficit-hyperactivity disorder as main manifestation. Deutsche Medizinische Wochenschrift 2002127 1250-1252. (https://doi.org/10.1055/s-2002-32098)

9 Martinez Lopez AB, Moreno Navarro JC, Maldonado Belmonte MJ \& Roldan Martin MB. Resistance to thyroid hormone and attention deficit hyperactivity disorder. Anales de Pediatria 201378 274-275. (https://doi.org/10.1016/j.anpedi.2012.08.011)
10 Stein MA, Weiss RE \& Refetoff S. Neurocognitive characteristics of individuals with resistance to thyroid hormone: comparisons with individuals with attention-deficit hyperactivity disorder. Journal of Developmental and Behavioral Pediatrics 199516 406-411.

11 Moran C, Habeb AM, Kahaly GJ, Kampmann C, Hughes M, Marek J, Rajanayagam O, Kuczynski A, Vargha-Khadem F, Morsy M, et al. Homozygous resistance to thyroid hormone beta: can combined antithyroid drug and triiodothyroacetic acid treatment prevent cardiac failure? Journal of the Endocrine Society 20171 1203-1212. (https://doi.org/10.1210/js.2017-00204)

Received in final form 26 September 2018

Accepted 25 October 2018 